\title{
Dynamic Public Health Interventions Consistent With the Development of COVID-19 Epidemic: The Targeted Prevention and Control Guidelines in Mainland, China
}

\section{Xinlei Miao}

Capital Medical University

Zhiyuan Wu

Capital Medical University

Chen Qiao

Capital Medical University

Mengmeng Liu

Capital Medical University

Zhiwei Li

Capital Medical University

Yijie Wang

Capital Medical University

Zongkai Xu

Capital Medical University

\section{Xiuhua Guo}

Department of Epidemiology and Health Statistics,School of Public health,Capital Medical University,Beijing,China https://orcid.org/0000-0001-6657-6940

Qun Meng ( $\nabla$ mengqun@nhfpc.gov.cn)

National health commission China

Research article

Keywords: COVID-19, Public health intervention, Effective reproduction number, Dynamic measures

Posted Date: August 13th, 2020

DOl: https://doi.org/10.21203/rs.3.rs-55959/v1

License: (c) (1) This work is licensed under a Creative Commons Attribution 4.0 International License. Read Full License 

Title: Dynamic Public Health Interventions Consistent With the Development of

COVID-19 Epidemic: The Targeted Prevention and Control Guidelines in Mainland,

China

\section{Authors:}

Xinlei Miao, ${ }^{1+2+}$ miaoxinlei1030@163.com;

Zhiyuan $\mathrm{Wu}^{1,2+}$ : wuxiaozhi@,ccmu.edu.cn;

Chen Qiao': 18591202366@163.com;

Mengmeng Liu ${ }^{1,2}$ as15999006637@163.com;

Zhiwei Li ${ }^{1,2}: \underline{15128472546 @ 163 . c o m ;}$

Yijie Wang ${ }^{1}$ wyj106122@,163.com;

Zongkai Xu1: xuzongkai1997@163.com;

Xiuhua Guo ${ }^{1,2 *}$ statguo@,ccmu.edu.cn;

Qun Meng ${ }^{1,3 *}$ mengqun@,nhfpc.gov.cn.

\section{Authors' affiliations:}

1. School of Public Health, Capital Medical University, Beijing, China;

2. Beijing Municipal Key Laboratory of Clinical Epidemiology, Beijing, China; 
3. National Health Commission of the People's Republic of China, Beijing, China.

First authors ${ }^{+}$: Xinlei Miao and Zhiyuan Wu drafted the manuscript together.

\section{Corresponding Author*:}

1. Qun Meng, National Health Commission of the People's Republic of China, No.38

Beilishilu Jia, Xicheng District, Beijing 100044, China. Tel: +86-010-68791226;

Email: mengqun@nhfpc.gov.cn;

2. Xiuhua Guo, Department of Epidemiology and Health Statistics, School of the

Public Health, Capital Medical University, No.10 Xitoutiao, Youanmen Street, Beijing,

100069, China. Tel: +86-010-83911508; Email: statguo@ccmu.edu.cn. 


\section{Abstract}

Background: This study aims to describe the dynamic characteristics of COVID-19 transmission and the public health interventions in three phases in mainland, China.

Methods: The number of daily reported new confirmed cases, severe cases and asymptomatic infected cases from Jan 10 to Jul 10 was analyzed. We calculated the effective reproduction number $(\mathrm{Rt})$ to reflect the dynamic characteristics of epidemic transmission and intervention effect. According to the overall guidelines for prevention and control, we divided the past six months into three phases and summarized the features of main public health interventions in each phase.

Results: The daily confirmed cases and severe cases of COVID-19 mainly concentrated in the first phase and the maximum Rt reached 10.75 (95\%CI: 10.26-11.24). With the society-wide efforts and joint prevention and control strategy, Rt began to decline below 1.0 from Feb 19. In the second phase, the occurrence of imported infected cases caused small fluctuations. The preventive strategy, preventing both imported cases and local spread of epidemic, was mainly taken. In the third phase, the government adopted policies to prevent imported cases and domestic 
re-infections, responding to the regular epidemic prevention demands.

Conclusion: Social isolation, wearing masks, digital management based on community and area hierarchical control were effective public health interventions in consistent with the development of COVID-19 epidemic. The targeted dynamic interventions in different phases could provide reference for other countries and regions to deal with COVID-19.

Keywords: COVID-19; Public health intervention; Effective reproduction number;

Dynamic measures

\section{What is new}

\section{Evidence before this study}

The public health interventions were effective for controlling the ongoing COVID-19 epidemic with approximately 88 thousand confirmed cases and 4.6 thousand deaths in China at the time of writing. Previous studies described the epidemiological characteristics of COVID-19 in the early stage and evaluated public health interventions in Wuhan where the outbreak happened. We found no article described 
the development course of COVID-19 epidemic in a long period and analyzed the dynamic features of the public health interventions in China.

\section{Added value of this study}

This study divided the past six months against the COVID-19 into three phases and summarized the dynamic public health interventions in each phase and estimated the effective reproduction number to reflect characteristics of the COVID-19 transmission and intervention effect. We proposed that the dynamic and targeted preventive measures should be adopted in different phases as the COVID-19 epidemic would be difficult to eliminate without vaccine and specific medicine. Regular epidemic prevention and control is required in China and other regions in the current stage.

\section{Implications of all the available evidence}

The results of this study provide reference for the policy maker in China and other countries that the public health interventions should be dynamic and updated as the development of COVID-19 epidemic, given the epidemiological characteristics, economic status and social impact. 


\section{Background}

Up to 10 July 2020 , there were nearly five millions confirmed cases and 0.6 millions deaths reported worldwide, indicating a high transmissibility and lethality of Coronavirus disease 2019 (COVID-19). The World Health Organization (WHO) appealed that countries and regions should adopt active interventions to prevent the transmission, treat infected cases and minimize the undesirable impact. ${ }^{1}$ In China, infected cases were first detected in Wuhan, Hubei Province in December 2019, ${ }^{2}$ followed by an acute human-to-human transmission. Worse still, the approaching time point of the Spring Festival made the epidemic control more difficult due to the mass population migration, which caused a large-scale outbreak. ${ }^{3}$

In general, the transmission came to a temporary end after a series of public health interventions, and no local case was ever reported from Mar $18,{ }^{4}$ followed by canceling the lockdown of Wuhan on Apr 8. Around March, the imported cases emerged and caused a threat for the epidemic control, which concentrated in Suifenhe City of Heilongjiang Province and Shulan City of Jinlin Province. From this point, the emphasis fell on the restriction and treatment of imported cases, which brought out a 
well controlled situation. However, the epidemic rebounded in Beijing from Jun 11 and it took 38 days for the risk degrading from public health response second level to third. ${ }^{5}$ Until now, some studies have described the epidemiological characteristics of COVID-19 in the early stage and evaluated the public health interventions in Wuhan. ${ }^{6,7}$ However, there were few studies analyzing the dynamics changes of COVID-19 transmission characteristics and the intervention features in different epidemic phases during the whole course.

In this study, we divided the whole epidemic course into three phases and analyzed the changes of COVID-19 transmission characteristics and the dynamic corresponding public health interventions from Jan 10 to Jul 10 in mainland, China.

\section{Methods}

\section{Sources of data}

The data of daily new confirmed cases, severe cases and imported cases from Jan 10 to Jul 10 in mainland of China was obtained from National Health Commission of the People's Republic of China (NHC). ${ }^{8}$ The data of daily new confirmed cases, severe cases in Hubei Province and Wuhan City was obtained from Health Commission of 
Hubei Province ${ }^{9}$ and Wuhan Municipal Health Commission, ${ }^{10}$ respectively. The public health measures were obtained from the relevant departments of China which were publicly available. ${ }^{11}$ The COVID-19 has been included in the national statutory infectious disease reporting system from Jan 20, which would be reported through the direct network reporting system within 2 hours once the diagnosis confirmed according to the unified national diagnostic standards. Therefore, it avoided the problem of missing data and false positive cases in the data largely, which ensured the reliability of results.

\section{Division of the three epidemic phases}

In order to summarize and describe the characteristics of COVID-19 epidemic and dynamic features of the interventions, we divided the past six months into three phases in accordance with the overall guidelines for prevention and control adopted by Chinese government. The first phase was from Jan 10 to Mar 3 with Society-wide efforts to prevent and control and the Joint Prevention and Control. Mar 4 to Jun 10 was considered to be the second phase, preventing both imported cases and local spread of the epidemic, mainly based on a series of non-pharmaceutical interventions. 
And the last phase was after Jun 11 in which the epidemic rebounded in a small range in Beijing. Preventing imported cases and domestic re-infections became the main guideline in this phase as the regular epidemic intervention demand.

\section{Case definition}

The definition of new cases and severe cases was based on the guidelines on diagnosis and treatment for pneumonia caused by novel coronavirus published by

National Health Commission of People's Republic of China. ${ }^{12}$ Asymptomatic infection case was defined according to the guidelines on prevent and control for pneumonia caused by novel coronavirus published by NHC (The detailed information of case definition shown in Supplementary material). ${ }^{13}$

\section{Statistical analysis}

We described the development trend of daily new confirmed cases, daily severe cases and asymptomatic infected cases. And Beijing was taken as an example to describe the policies and measures in regular epidemic prevention and control phase.

We summarized the main public health interventions after COVID-19 outbreak in three phases, respectively (The detailed information of interventions shown in 
Supplementary material). The transmission characteristics of COVID-19 was analyzed using the effective reproduction number (Rt). The number of local daily new confirmed cases and the serial interval (assuming a Gamma distribution with mean $7 \cdot 5$ days, standard deviation 3.4 days $^{14}$ ) were used to estimate Rt and its $95 \%$ confidence interval (CI) on each day via a seven-day moving shift window. The Rt was also estimated when imported cases considered. All the analysis was performed using packages 'ggplot2' and 'EpiEstim' in R software (version 3·6·3).

\section{Results}

\section{The dynamic characteristics of COVID-19}

A total of 83,587 confirmed cases were reported from Jan 10, 2020 to Jul 10, 2020 in mainland of China as shown in Figure 1. Most of the confirmed cases concentrated in the first phase. Imported cases occurred in the second phase, and the number of daily confirmed cases fluctuated in a small extent. The similar epidemic trend was seen in Hubei Province and Wuhan City. There were two peaks in new confirmed cases on

Feb 12 and Apr 16 (The detailed information of Rt estimation shown in Supplementary material). After the epidemic was gradually brought under control, 
the rebounded in Beijing broke the situation of no new confirmed cases in the past 26

days, with a local case reported in the Xinfadi market on Jun 11. From this point, the epidemic stepped into the third phase.

As shown in Figure 2, daily change of severe cases gradually increased since Jan 21.

Most severe cases concentrated in the first phase. It was worth noting that the number

of severe cases in Hubei Province was more than in the whole country on Feb 12 (The

explanation of the peaks shown in Supplementary material). In the second and third phases, the number of daily severe cases declined. The daily change of severe cases in Hubei Province and Wuhan City showed similar trend. Asymptomatic infections emerged from the latter period of the second phase. The overall status of asymptomatic infection was controlled with occasional fluctuations.

\section{Changes of Rt estimation among three phases}

As shown in Figure 3 (A), Rt increased sharply in the first period, which reached a maximum of $10 \cdot 74(95 \% \mathrm{CI}: 10 \cdot 26-11 \cdot 24)$ on Jan 25 and fell below the controlled level (Rt: 0.58 ; 95\%CI: 0.58-0.59) from Feb 19. In the second period, the Rt fluctuated around 1.00 and the overall level was below 1.00 as shown in Figure 3 (C). 
In the third period, the peak (Rt: 5.67; 95\%CI: 4.95-6.45) appeared on Jun 16 and remained controlled from Jun 23 (Rt: 0.91; 95\%CI: 0.77-1.05). In addition, the Rt was estimated given the daily imported case considered as shown in Figure 3 (B) and Figure 3 (D). The detailed information of Rt estimation was shown in Supplementary material.

\section{Regular prevention and targeted control in Beijing}

From Jun 11, the epidemic rebounded in Beijing and the targeted control strategy by stratifying the risk level of community was proposed. Four regions of middle-risk appeared on Jun 13 and the first high-risk region arose on Jun 14. Then, other regions were defined and regulated as middle-high risk one after another, among which there were 39 middle-risk regions at most and five high-risk regions. From Jun 29, the amount of middle-risk regions began to decrease and the number of high-risk regions declined from Jun 29. The distribution of daily risk regions in Beijing from Jun 11 to Jul 10 was shown in Figure 4.

\section{Discussion}

This study analyzed the dynamic characteristics of COVID-19 transmission and the 
public health interventions in the past six months in mainland, China. Dr. An Pan evaluated the public health interventions by dividing the epidemic period into five main stages to discuss the association of the COVID-19 epidemic and the public health interventions. ${ }^{6}$ Our study divided the whole period according to the epidemic prevention and control guidelines, which could highlight the dynamic changes of public health interventions and the remarkable prevention and control effects. The decreased Rt and the declined number of daily new confirmed cases indicated that the guidelines by Chinese government were effective in controlling the COVID-19 epidemic.

After the COVID-19 outbreak, the government immediately restricted the traffic in Wuhan and performed hierarchical traffic control in non-severely affected areas. ${ }^{15}$ The government adopted classified isolation treatment for infected cases. To implement the guidelines of 'treating all who need to be hospitalized and treated, the confirmed cases and severe cases were admitted to the designated hospitals, while mild patients were arranged to be treated in Fangcang hospital, a temporary isolation warehouse, ${ }^{16}$ and the fever patients, suspected patients and close contacts were all quarantined for 
centralized observation. In policy review, we found that the Chinese government repeatedly emphasized the prohibition of gathering activities and wearing masks in its successively updated prevention and control guidelines and nationally issued policies. Other studies also showed that under the premise of policy intervention, physical measures effectively reduced the spread of infectious diseases. ${ }^{17-19}$ These interventions showed that in China, such a super-large population mobile society, physical measures were the most important measures to curb the spread of the epidemic such as unprecedented social isolation and mask protection, which played a key role in the situation without vaccines and special medicines.

When we deeply understood the policy that society-wide efforts to prevent and control, we discovered that the Chinese government took a measure called pull-net investigation to implement the home symptom investigation action of leaving no one unscreened, which was called community prevention and control. ${ }^{20}$ During the outbreak, Wuhan dispatched more than 80,000 community officials to form 1,800 teams to conduct epidemiological investigation on four million residents ${ }^{21}$. With the spread of the epidemic, four million community workers with the cooperation of 
volunteers visited 650,000 communities across the country, ${ }^{22}$ based the digital management for a matrix of communities to investigate the trajectories of infected persons thoroughly. Epidemiological investigation is a key link in tracking the infected and cutting off the chain of transmission. Strictly implement the source prevention and the community prevention and control of early detection, early reporting, early isolation and early treatment, ${ }^{23}$ which play a vital role in reducing the rate of infection and mortality, and providing important support for prevention and control forces.

According to the statistics, more than 42,600 medical workers were dispatched from the hospitals or community of all over the country to support Hubei since Jan 23, Chinese Lunar New Year in order to relieve the medical burden on treatment in Wuhan. ${ }^{24,25}$ Therefore, the unified command and leadership of the government is the necessary strength to mobilizing the whole society forces and medical personnel and medical supplies for preventing, controlling and treating.

Although it was a small-scale rebound of the epidemic, with the continuous spread of the global epidemic, the world may be in a state of epidemic prevention and control 
for a long time, which was defined as regular epidemic ${ }^{26}$. We concluded that in the face of regular control, the Chinese government adopted strict guidelines of preventing imported cases, local spread of the epidemic and domestic re-infections, which showed remarkable effect. The study found that the Rt increased slightly with imported cases from Suifenhe City of Heilongjiang province and Shulan City of Jilin province, while quickly returned below the controlled level. There was an rebound of local cases in Beijing, and the Rt rose rapidly on June 11, but it quickly dropped to below 1.00 just after 12 days. The different measures were taken in response to different situations to prevent the epidemic from spreading on a large scale. For example, the first point of entry mechanism was adopted to cut off the spread of imported cases, ${ }^{27}$ and epidemiological investigations with different focuses have been carried out to respond to the situation of asymptomatic infections, and residents were required and encouraged to carry out nucleic acid testing so as to realize test all who need and want to be tested. In response to the fluctuations in Beijing, it divided the area into three risk levels of high, medium and low to adopted different strategies according to the number of confirmed cases. ${ }^{28}$ These important interventions could 
provide valuable experience for the global to deal with the regular epidemic prevention and control.

This study had several limitations. First, there existed the lag effect between the daily reported confirmed numbers and the real infected numbers due to the inaccessibility of detect capability, which could slightly bias the descriptive results and Rt estimation. Second, we artificially divided the whole epidemic course into three phases and explored the transmission characteristics and prevention strategies in China. The definition of the three phases was relatively limited and the development of COVID-19 in other countries may not follow this dynamic trajectory as in China.

\section{Conclusion}

In the past six months against the COVID-19 in China, the interventions taken in each phase were adjusted such as social isolation, mask protection, digital management based on community, and area hierarchical control, which were important for preventing and controlling the epidemic. These dynamic interventions in different epidemic phases could provide references for the global in response to the regular epidemic demand. 


\section{Declarations}

\section{Ethics approval and consent to participate}

The study was approved by Ethics Committee of Capital Medical University.

\section{Competing interests}

The authors declare that they have no conflicts of interest.

\section{Funding}

Not applicable.

\section{Authors' contributions}

MXL and WZY wrote the manuscript. XZK and WYJ collected the data. QC, LMM and LZW performed the analysis and interpretation. MQ and GXH designed the study.

All authors have read and approved the manuscript.

\section{Data Availability}

All data used within the study is publicly available. 


\section{References}

1. WHO. Coronavirus disease (COVID-19) Pandemic. https://www.who.int/emergencies/diseases/novel-coronavirus-2019/Accessed on $11 \mathrm{Jul} 2020$.

2. CCTV. China publishes timeline on COVID-19 information sharing, int'l cooperation

http://english.cctv.com/2020/04/07/ARTIL1AoiiBZ12km6vLzKTNg200407.shtm 1?spm=C69523.P89571092934.E19742616158.1. /Accessed on 11 Jul 2020.

3. Zhu N, Zhang D, Wang W, et al. A Novel Coronavirus from Patients with Pneumonia in China, 2019. N Engl J Med 2020; 382(8): 727-33.

4. Huanqiu.com. For the first time in mainland China, there are no new suspected local cases. 2020/03/18. https://www.sohu.com/a/381085322_162522. /Accessed on 13 Jul 2020.

5. cctv.com. The emergency response level in Beijing was adjusted to the second level. 2020. https://baijiahao.baidu.com/s?id=1669707499046495319\&wfr=spider\&for=pc /Accessed on 02 Aug 2020.

6. Pan A, Liu L, Wang C, et al. Association of Public Health Interventions With the Epidemiology of the COVID-19 Outbreak in Wuhan, China. JAMA 2020.

7. Wu JT, Leung K, Leung GM. Nowcasting and forecasting the potential domestic and international spread of the 2019-nCoV outbreak originating in Wuhan, China: a modelling study. The Lancet 2020; 395(10225): 689-97. 
8. NHC. National Health Commission of the People's Republic of China. http://www.nhc.gov.cn. /Accessed on 12 Jul 2020.

9. Province HCoH. Health Commission of Hubei Province. 2020. http://wsbs.hbwsjs.gov.cn/hbwsjsMobile/index.html /Accessed on 12 Jul 2020.

10. Commission WMH. Wuhan Municipal Health Commission. 2020. http://wjw.wuhan.gov.cn/Accessed on 12 Jul 2020.

11. www.gov.cn. Policy Document Bank of the State Council. http://www.gov.cn.

12. NHC. The seventh version of guidelines on diagnosis and treatment for pneumonia caused by novel coronavirus, 2020 .

13. NHC. The sixth version of guidelines on prevent and control for pneumonia caused by novel coronavirus, 2020.

14. Li Q, Guan X, Wu P, et al. Early Transmission Dynamics in Wuhan, China, of Novel Coronavirus-Infected Pneumonia. 2020; 382(13): 1199-207.

15. Huanqiu.com. Request traffic control. 2020. https://baijiahao.baidu.com/s?id=1659044586443705492\&wfr=spider\&for=pc. /Accessed on 16 Jul 2020.

16. Chen S, Zhang Z, Yang J, et al. Fangcang shelter hospitals: a novel concept for responding to public health emergencies. Lancet 2020; 395(10232): 1305-14.

17. Markel H, Lipman HB, Navarro JA, et al. Nonpharmaceutical interventions implemented by US cities during the 1918-1919 influenza pandemic. JAMA 2007; 298(6): 644-54.

18. Poletti P, Caprile B, Ajelli M, Pugliese A, Merler S. Spontaneous behavioural 
changes in response to epidemics. J Theor Biol 2009; 260(1): 31-40.

19. Chen S, Yang J, Yang W, Wang C, Barnighausen T. COVID-19 control in China during mass population movements at New Year. Lancet 2020; 395(10226): 764-6.

20. NHC. Precise and refined guidelines for community prevention, control and $\begin{array}{llll}\text { service } & \text { of } & \text { COVID-19. }\end{array}$ http://www.nhc.gov.cn/jws/s7874/202004/8bc0fde9ef6e4820a30b546f396698c0. shtml. /Accessed on 17 Jul 2020.

21. Wuhan Pi. More than 800 epidemiological investigation teams investigate the $\begin{array}{lll}\text { epidemic } & \text { in } & 2020 .\end{array}$ https://baijiahao.baidu.com/s?id=1662053454078734705\&wfr=spider\&for=pc. /Accessed on 17 Jul 2020.

22. NHC. 4 million community workers visited 650,000 communities across China. 2020. http://www.gov.cn/xinwen/2020-06/07/content_5517738.htm. /Accessed on 20 Jul 2020.

23. NHC. Director Ma Xiaowei delivered a speech at the 73rd World Health Assembly. 2020. http://www.nhc.gov.cn/wjw/xwdt/202005/1d72686f7f2f41b9900e79486bd8ab92. shtml. /Accessed on 20 Jul 2020.

24. NHC. The text of the press conference on April 1, 2020. http://www.nhc.gov.cn/xcs/s3574/202004/73b1216f249f4498929eed592afe51fb. shtml. /Accessed on 20 Jul 2020. 
25. NHC. The State Council Information Office March 31, 2020 press conference text

record.

2020.

http://www.nhc.gov.cn/xcs/yqfkdt/202003/26403157baf8418292f3f69110af1d1e. shtml /Accessed on 20 Jul 2020.

26. www.gov.cn. The State Council's Joint Prevention and Control Mechanism issued the "Guiding Opinions on Doing a Good Job in the regular epidemic of the New Coronary Pneumonia Prevention and Control Work". 2020. http://www.gov.cn/xinwen/2020-05/08/content 5509965.htm. /Accessed on 26 Jul 2020.

27. NHC. Notice on Doing a Good Job in the Detection of New Coronavirus in Cities Related to Preventing the Import of Epidemics. 2020. http://www.nhc.gov.cn/yzygj/s7659/202004/9c1b7e72557447f68927423d3012f1 68.shtml. /Accessed on 26 Jul 2020.

28. NHC. the fifth guidelines on prevent and control for pneumonia caused by novel coronavirus, 2020. /Accessed on $30 \mathrm{Jul} 2020$. 


\section{Figures:}

Figure 1: Epidemic trends of daily new confirmed cases, main guidelines and public health interventions during the three phases of COVID-19 epidemic in mainland,

China from Jan 10 to Jul 10.

Figure 2: Epidemic trends of daily change of severe cases and asymptomatic infections in mainland, China from Jan 10 to Jul 10.

Figure 3: The estimation of daily Rt and the average level of three epidemic phases from Jan 10 to Jul 10, 2020.

Legend:

A: Changes of daily Rt estimated by number of local daily reported case; B: Rt average level of three epidemic periods estimated by number of local daily reported case; C: Changes of daily Rt estimated by number of local and imported daily reported case; D: Rt average level of three epidemic period estimated by number of local and imported daily reported case.

Figure 4: The daily distribution of middle and high risk regions in Beijing from Jun 11 to Jul 10. 


\section{Figures}

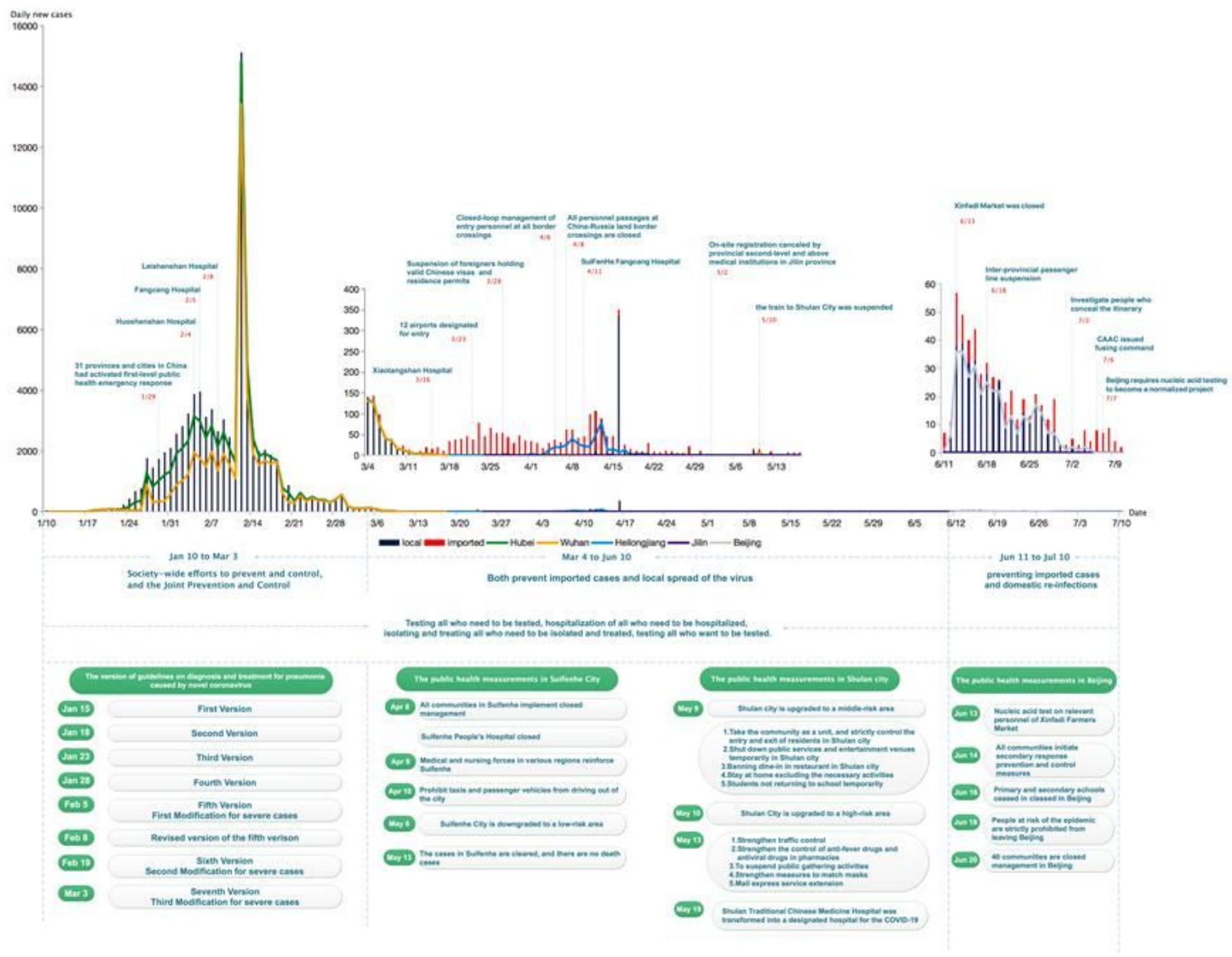

Figure 1

Epidemic trends of daily new confirmed cases, main guidelines and public health interventions during the three phases of COVID-19 epidemic in mainland, China from Jan 10 to Jul 10. 


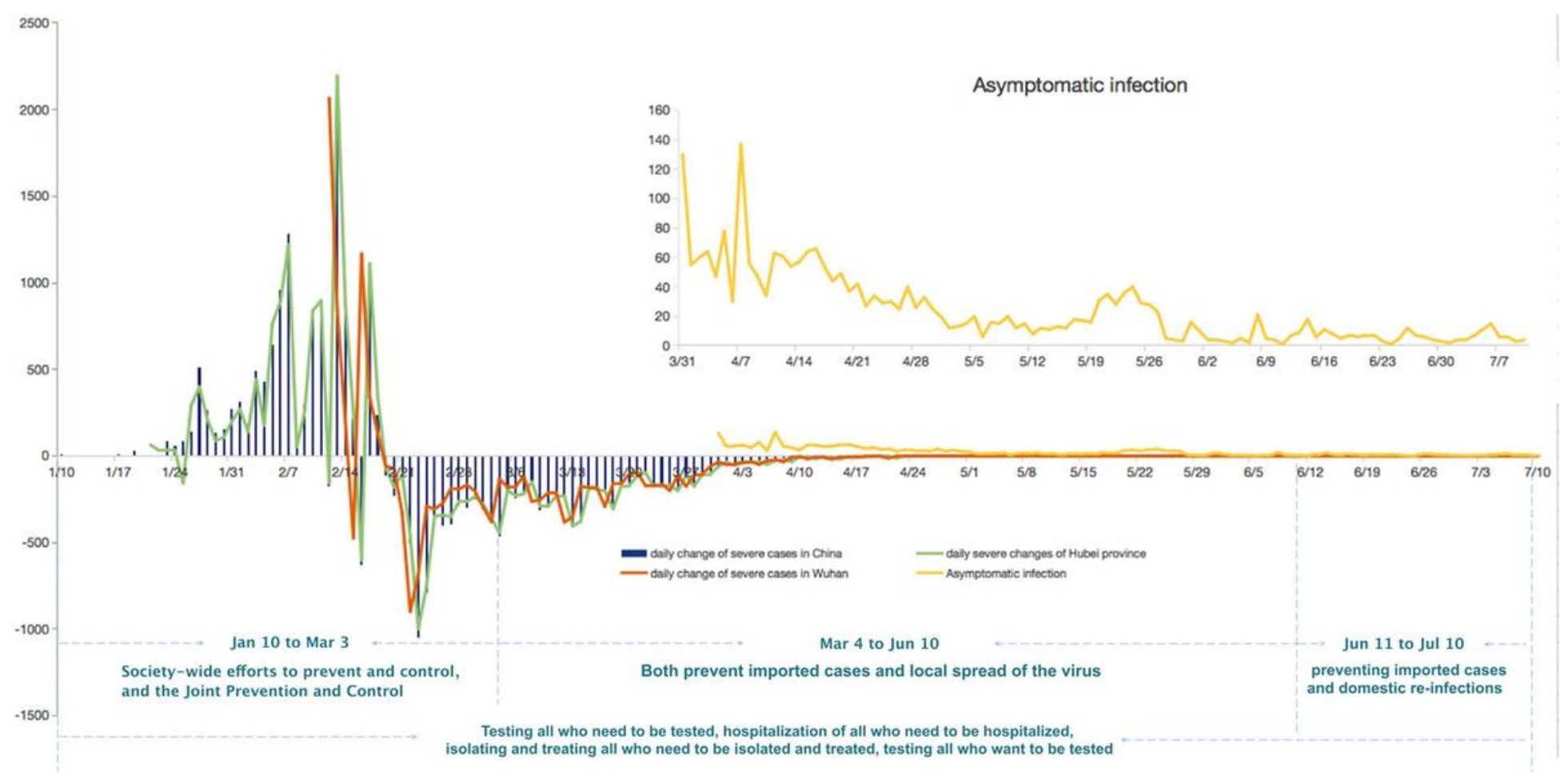

Figure 2

Epidemic trends of daily change of severe cases and asymptomatic infections in mainland, China from Jan 10 to Jul 10.

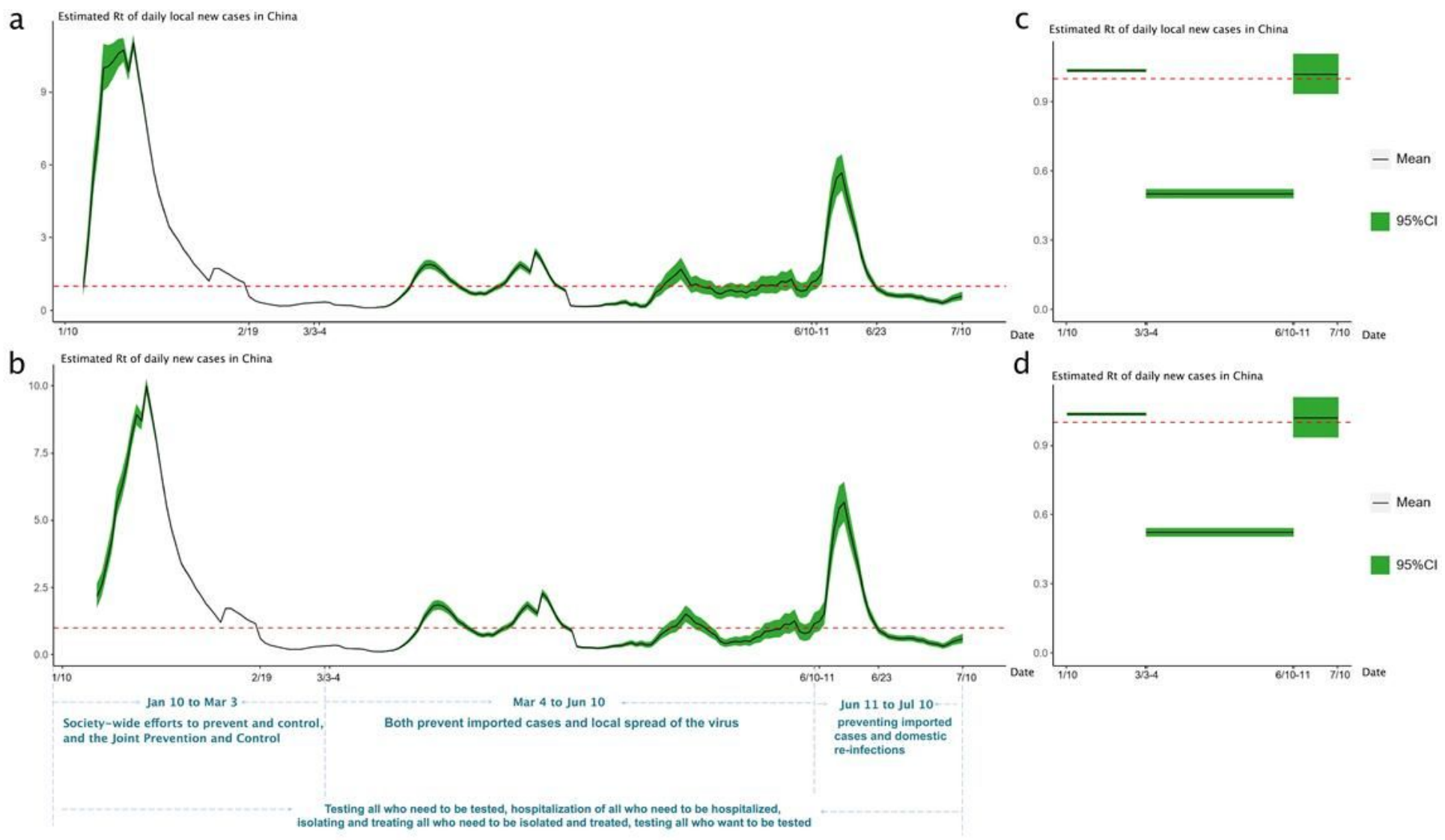

Figure 3 
The estimation of daily Rt and the average level of three epidemic phases from Jan 10 to Jul 10, 2020. Legend: A: Changes of daily Rt estimated by number of local daily reported case; B: Rt average level of three epidemic periods estimated by number of local daily reported case; $\mathrm{C}$ : Changes of daily Rt estimated by number of local and imported daily reported case; D: Rt average level of three epidemic period estimated by number of local and imported daily reported case.

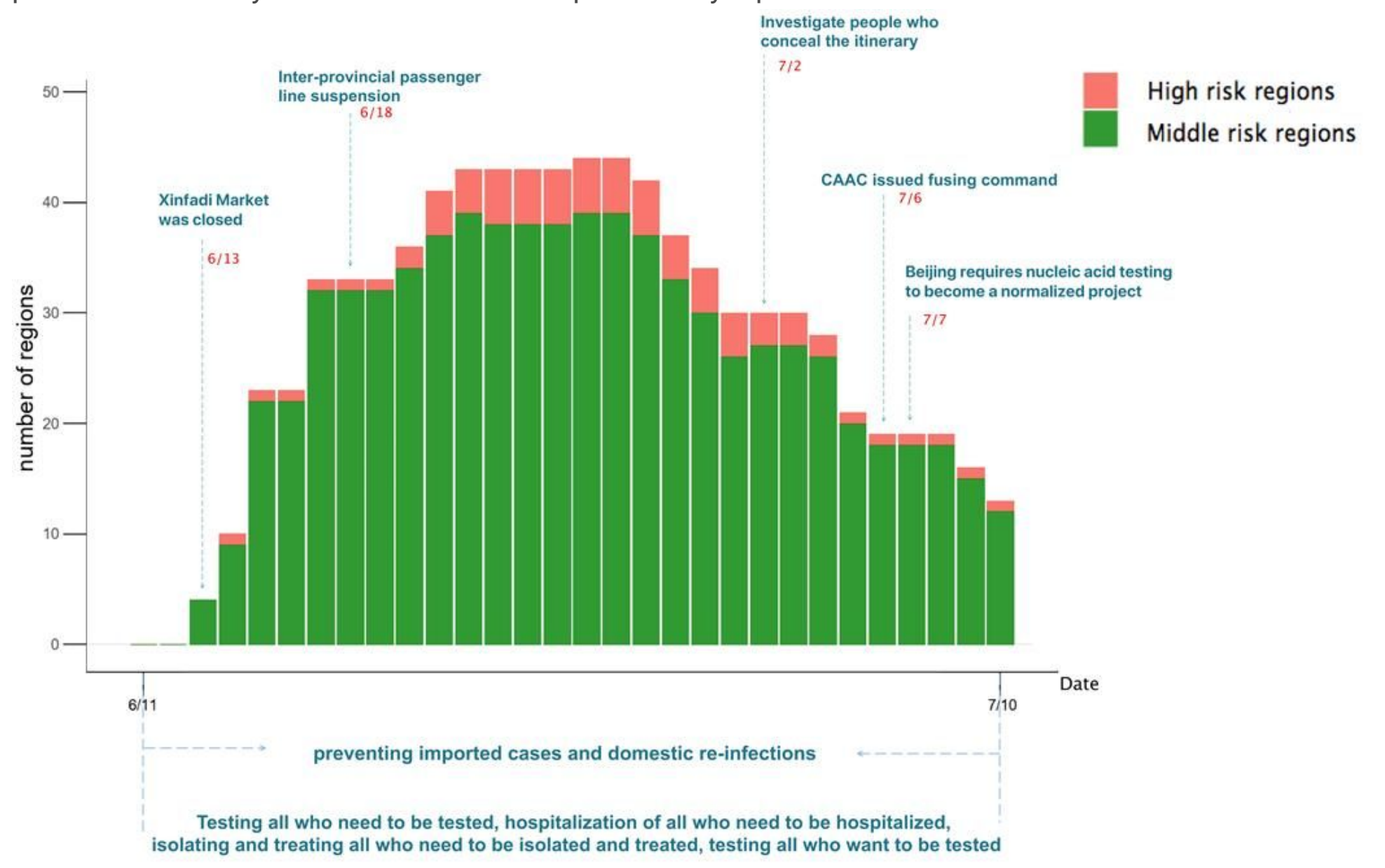

\section{Figure 4}

The daily distribution of middle and high risk regions in Beijing from Jun 11 to Jul 10.

\section{Supplementary Files}

This is a list of supplementary files associated with this preprint. Click to download.

- Supplementarymaterial.pdf 